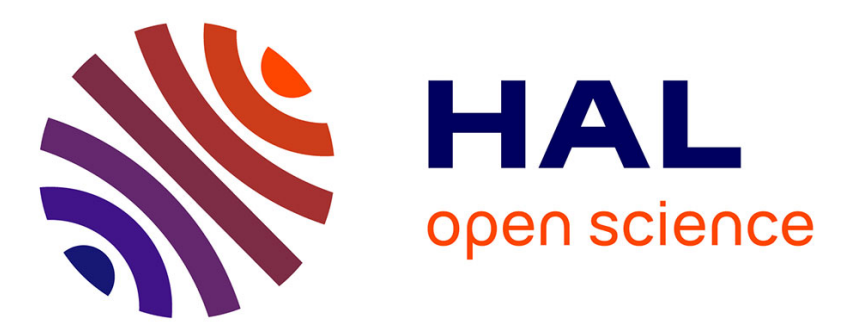

\title{
Analyses of heated concrete spalling due to restrained thermal dilation: Application to the "Chunnel" fire
} Y. Msaad, Guy Bonnet

\section{To cite this version:}

Y. Msaad, Guy Bonnet. Analyses of heated concrete spalling due to restrained thermal dilation: Application to the "Chunnel" fire. Journal of Engineering Mechanics - ASCE, 2006, 132 (10), pp.11241132. 10.1061/(ASCE)0733-9399(2006)132:10(1124) . hal-00691112

\section{HAL Id: hal-00691112 https://hal.science/hal-00691112}

Submitted on 16 Jan 2016

HAL is a multi-disciplinary open access archive for the deposit and dissemination of scientific research documents, whether they are published or not. The documents may come from teaching and research institutions in France or abroad, or from public or private research centers.
L'archive ouverte pluridisciplinaire HAL, est destinée au dépôt et à la diffusion de documents scientifiques de niveau recherche, publiés ou non, émanant des établissements d'enseignement et de recherche français ou étrangers, des laboratoires publics ou privés. 


\title{
Analyses of Heated Concrete Spalling due to Restrained Thermal Dilation: Application to the "Chunnel" Fire
}

\author{
Y. Msaad ${ }^{1}$ and G. Bonnet ${ }^{2}$
}

\begin{abstract}
Two spalling mechanisms are generally quoted in the literature. The first one is due to pore pressure buildup. The second one, which is analyzed in this paper, is generated by restrained thermal dilation. We seek to model the channel tunnel fire by a thermochemoplastic constitutive model. A simplified analytical approach allows us to express mechanical variables as stresses and strains near the heated surface (the concrete wall). This study leads to introducing a "plastification" temperature and to deduce a plastification depth that can be useful for determining the spalling localization. A comparison between different cases (with and without chemical softening or decohesion) shows that thermal spalling is due to chemical decohesion (strength degradation) and not to chemical softening (rigidity reduction).
\end{abstract}

CE Database subject headings: Concrete; Thermal factors; Spalling.

Introduction

The material and structural behavior of concrete at high temperature is well described in the literature (Anderberg 1997; Bazant 1997). The channel tunnel fire revealed, however, the lack of understanding of the mechanisms that lead to concrete member spalling, which beside logistic questions concerning safety, generates economic losses at the reparation moment.

This study seeks to evaluate the effects of thermal softening $E$ and thermal decohesion $f_{c}$ (due to dehydration) upon the compressive stresses generated by the restrained thermal dilation. In a first step, the principle of the dehydration effects on the concrete mechanical properties is presented. Next, equations for the thermoelastoplastic model (Ulm et al. 1999b) are given. Then, a model application for the "Chunnel" fire is present, based on an analytical approach using some simplifications that are justified and validated later on by the numerical complete model (without approximations). The simplified mechanical model obtained is very advantageous since its properties are related to a purely thermal problem. Finally, the ability of the simplified model to forecast the spalling formation is discussed while examining the combination of the two main phenomena: thermal softening and thermal decohesion.

${ }^{1}$ Professor, Institut Navier, Univ. de Marne la Vallée, 6, 8 ave. Blaise Pascal, Marne-la-Vallée, 77455 France (corresponding author). E-mail: y.msaad@cerib.com

${ }^{2}$ Institut Navier, Univ. de Marne la Vallée, 5, blvd. Descartes, Marne-la-Vallée, 77455 Cedex 2, France. E-mail: guy.bonnet@ univ-mlv.fr

Note. Associate Editor: Christian Hellmich. Discussion open until March 1, 2007. Separate discussions must be submitted for individual papers. To extend the closing date by one month, a written request must be filed with the ASCE Managing Editor. The manuscript for this paper was submitted for review and possible publication on December 30, 2004; approved on October 24, 2005. This paper is part of the Journal of Engineering Mechanics, Vol. 132, No. 10, October 1, 2006. (OASCE, ISSN 0733-9399/2006/10-1124-1132/\$25.00.

\section{Chemoplastic Model}

In this model, two chemical dehydration effects are considered.

\section{Chemical Softening}

Young's modulus of degradation is due to the decomposition of the cement connections. It can be supposed that rigidity is proportional to the remaining mass of hydrates. The proportionality has been found to constitute an intrinsic material property of concrete. Thus, the dehydration degree $\xi=m_{h} / m_{h 0}=$ relative ratio of rigidity after heating

$$
E=\xi E_{0}
$$

This dehydration degree describes a chemical reaction that has a chemical kinetic with a characteristic time $\tau_{\xi}$ and an asymptotic limit $\xi_{\text {eq }}(T)$ (Fasseu 1997), leading to

$$
\dot{\xi}=-\frac{1}{\tau_{\xi}}\left[\xi-\xi_{\mathrm{eq}}(T)\right]
$$

\section{Chemical Decohesion}

The dehydration also has an influence on the elastic limit strength that is again supposed to be proportional to the dehydration degree. Thus, the isotropic elastic limit criterion of WilliamWarnke (William and Warnke 1975) takes into account this chemical decohesion as follows:

$$
f(\sigma, \xi)=\tau+\delta(\Theta)\left(\sigma-\rho_{0} \xi\right)
$$

where the three invariants are $\tau=\sqrt{1 / 2 s: s}, \quad \sigma=(1 / 3) \operatorname{tr}(\sigma)$, and $\cos (\Theta)=\left(2 \sigma_{1}^{\mathrm{pr}}-\sigma_{2}^{\mathrm{pr}}-\sigma_{3}^{\mathrm{pr}}\right) / \sqrt{12} \tau$; and where $s=$ stress deviator; $\rho_{0}=$ cohesion pressure before dehydration; and $\delta(\Theta)=$ friction angle, which depends on Lode angle $\Theta$, defined above as follows:

$$
\delta(\theta)=\frac{u+v}{w}
$$

where 


$$
\begin{gathered}
u=2 \delta_{c}\left(\delta_{c}^{2}-\delta_{t}^{2}\right) \cos (\theta) \\
v=\delta_{c}\left(2 \delta_{t}-\delta_{c}\right) \sqrt{4\left(\delta_{c}^{2}-\delta_{t}^{2}\right) \cos ^{2}(\theta)+5 \delta_{t}-4 \delta_{c} \delta_{t}} \\
w=4\left(\delta_{c}^{2}-\delta_{t}^{2}\right) \cos ^{2}(\theta)+\left(\delta_{c}-2 \delta_{t}\right)^{2}
\end{gathered}
$$

Finally, the friction coefficients $\delta_{c}$ and $\delta_{t}$ and the cohesion pressure $\rho_{0}$ are related to the standard properties (ultimate compressive strength $f_{c}$, ultimate tensile strength $f_{t}$, and ultimate bicompressive strength $f_{\mathrm{bc}}$ ) by

$$
f_{c}=\frac{3 \delta_{c}}{\sqrt{3}-\delta_{c}} \rho_{0} ; \quad f_{t}=\frac{3 \delta_{t}}{\sqrt{3}+\delta_{t}} \rho_{0} ; \quad f_{\mathrm{bc}}=\frac{3 \delta_{t}}{\sqrt{3}-2 \delta_{t}} \rho_{0}
$$

\section{Comparison between Thermal and Chemical Characteristic Times}

Neglecting both elastic deformation latent heat and energy dissipation, the heat balance equation reads (in an unidirectional case with Fourier's law)

$$
C \dot{T}-\kappa \nabla_{x}^{2} T=0
$$

where $C$ and $\kappa=$ volumic heat capacity and conductivity of concrete. The characteristic time of heat conduction [described in Eq. (4)] is then related to the characteristic length $L$ by $\tau_{T}=\left(\kappa L^{2}\right) / C$.

The characteristic time $\tau_{\xi}$ of dehydration [described in Eq. (2)] can be roughly estimated by considering the microdiffusion between the micropores and the capillary pores, which occurs faster when temperature is higher [because of the activation Arrhenius term $\left.\exp \left(E_{a} / R T\right)\right]$ and then $\tau_{\xi}(T) \leqslant \tau_{\xi}\left(T_{0}\right) \approx 30 \mathrm{~s}$.

Neglecting $\tau_{\xi}$ with respect to $\tau_{T}$ is possible when $L \gg \sqrt{\tau_{\xi}\left(T_{0}\right)_{\kappa} / C} \approx 5 \mathrm{~mm}$, which is always verified. Then, it can be supposed that $\tau_{\xi} \ll \tau_{T}$.

Thus, the dehydration degree $\xi$ given in Eq. (2) can be supposed equal to its asymptotic value $\left[\xi=\xi_{\text {eq }}(T)\right.$ at each time]. Thus, and according to Eq. (1), Young's modulus $E(\xi)$ reads $E(T)$.

\section{Thermoelastoplastic Deformation}

The total strain is the sum of elastic, plastic, and thermal strains

$$
\varepsilon^{\mathrm{tot}}=\varepsilon^{\mathrm{th}}+\varepsilon^{\mathrm{el}}+\varepsilon^{\mathrm{pl}}
$$

\section{Thermal Strain}

The thermal dilation is characterized by a thermal strain tensor $\alpha$. It is assumed in the following that the medium is isotropic so that $\alpha=\alpha 1$ and then

$$
d \varepsilon^{\text {th }}=\alpha d T 1
$$

\section{Elastic Strain}

The elastic strain tensor is related to the stress tensor via the four-order elastic stiffness tensor $\hat{C}$ as follows:

$$
d \sigma=\hat{C} d \varepsilon^{\mathrm{el}}
$$

where $\hat{C}=\xi \hat{C}_{0}$ is assumed to depend on the dehydration degree. This assumption is a generalized form of the result in Eq. (1).

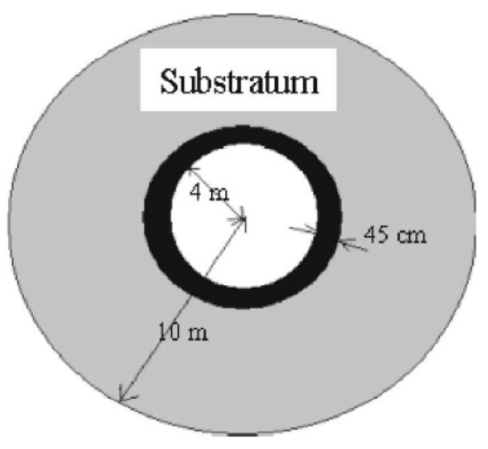

Fig. 1. Geometry of the channel tunnel

In the isotropic case, the elastic stiffness tensor of the last equation reads

$$
\hat{C}=K(T) 1 \otimes 1+2 G(T)\left(\hat{I}-\frac{1}{3} 1 \otimes 1\right)
$$

and then

$$
d \sigma=\left[K(T)-\frac{2}{3} G(T)\right] \operatorname{tr}\left(d \varepsilon^{\mathrm{el}}\right)+2 G(T) d \varepsilon^{\mathrm{el}}
$$

where $K(T)=[E(T) / 3(1-2 v)]=$ bulk modulus and $G(T)$ $=[E(T) / 2(1+v)]=$ shear modulus, assuming that the Poisson's ratio is constant.

\section{Plastic Strain and Flow Rule}

We suppose that the plasticity rule is associated. The plastic flow is, therefore, given by the relation

$$
d \varepsilon^{\mathrm{pl}}=d \lambda \frac{d f}{d \sigma}
$$

where $d \lambda=$ plastic multiplier, which is null only when the evolution is purely elastic $(f<0$ or $\dot{f}<0)$ and positive when there is a plastic flow $(f=\dot{f}=0)$.

\section{“Chunnel” Fire}

In this section, the thermochemomechanical behavior of the cylindrical channel tunnel heated during a fire (U1m et al. 1999a) will be studied.

\section{Geometry}

Fig. 1 presents the geometry of the channel tunnel. Due to the axisymmetric geometry, the geometric problem variable reduces to the radius $r$ from the tunnel center. The substratum external radius is large enough so that the boundary conditions on extrados do not influence the thermal and mechanical fields on intrados (concrete wall)

This axisymmetric geometry allows us to write the following helpful relations:

$$
\varepsilon_{r \theta}=\varepsilon_{r z}=\varepsilon_{\theta z}=\varepsilon_{z z}=0
$$


Table 1. Thermal and Mechanical Properties Used in Numerical Analysis

\begin{tabular}{|c|c|c|}
\hline Property & Concrete & Chalk \\
\hline Elastic Young's modulus $E_{0}$ & $42 \mathrm{GPa}$ & $4 \mathrm{GPa}$ \\
\hline Poisson's ratio $v$ & 0.2 & 0.25 \\
\hline $\begin{array}{l}\text { Ultimate compressive } \\
\text { strength } f_{c}\end{array}$ & $80 \mathrm{MPa}$ & - \\
\hline Ultimate tensile strength $f_{t}$ & $6.4 \mathrm{MPa}\left(=0.08 \cdot f_{c}\right)$ & - \\
\hline $\begin{array}{l}\text { Ultimate biaxial compressive } \\
\text { strength } f_{\mathrm{bc}}\end{array}$ & $88 \mathrm{MPa}\left(=1.1 \cdot f_{c}\right)$ & - \\
\hline Thermal dilation coefficient $\alpha$ & $10^{-5} \mathrm{~K}^{-1}$ & $10^{-5} \mathrm{~K}^{-1}$ \\
\hline Volumic heat capacity $C$ & $2.5 \times 10^{6} \mathrm{~J} \mathrm{~m}^{-1} \mathrm{~K}^{-1}$ & $2 \times 10^{6} \mathrm{~J} \mathrm{~m}^{-1} \mathrm{~K}^{-1}$ \\
\hline Conductivity $\kappa$ & $2.22 \mathrm{~J} \mathrm{~m}^{-1} \mathrm{~K}^{-1} \mathrm{~s}^{-1}$ & $1.94 \mathrm{~J} \mathrm{~m}^{-1} \mathrm{~K}^{-1} \mathrm{~s}^{-1}$ \\
\hline Convective heat exchange $h$ & $4.16 \mathrm{~J} \mathrm{~m}^{-2} \mathrm{~K}^{-1} \mathrm{~s}^{-1}$ & - \\
\hline
\end{tabular}

Note: Yield criterion is completely defined by $f_{c}, f_{t}$, and $f_{\mathrm{bc}}$

$$
\varepsilon_{r r}=\frac{\partial u_{r}}{\partial r}, \quad \varepsilon_{\theta \theta}=\frac{u_{r}}{r}
$$

and then

$$
\varepsilon_{r r}=r \frac{\partial \varepsilon_{\theta \theta}}{\partial r}+\varepsilon_{\theta \theta}
$$

The balance equation $\operatorname{div}(\sigma)=0$ simply reads in this case

$$
\sigma_{\theta \theta}=r \frac{\partial \sigma_{r r}}{\partial r}+\sigma_{r r}
$$

The heat conduction equation reads

$$
C \frac{\partial T}{\partial t}-\frac{1}{r} \kappa \frac{\partial}{\partial r}\left(r \frac{\partial T}{\partial r}\right)=0
$$

\section{Material Properties}

The tunnel concrete wall is modeled in thermochemoplasticity and the chalk substratum is treated in thermoelasticity. The material properties of the different constituents are given in Table 1.

\section{Boundary Conditions}

For this unidimensional model, two boundary conditions are needed to be fixed at $r=r_{\text {int }}$ and $r=r_{\text {ext }}$.

- Convection at intrados: $q=-\lambda(\partial T / \partial r)=h\left[T-T_{\text {imp }}(t)\right]$ at $r=r_{\text {int }}, \quad$ where $\quad T_{\text {imp }}(t)=T_{0}+1,280[1-0.325 \exp (-0.167 t)$ $-0.675 \exp (-2.5 t)]=$ temperature increase given by the Highway Capacity Manual curve (typical for tunnels) and where $t=$ time in minutes;

- Adiabatic exchange at extrados: $q=0$ at $r=r_{\text {ext }}$;

- Intrados free of any radial stress: $\sigma_{r r}=0$ at $r=r_{\text {int }}$

- Restrained radial displacement on extrados: $u_{r}=0$ at $r=r_{\text {ext }}$ $\left[\varepsilon_{\theta \theta}=0\right.$ according to Eq. (10)].

\section{Thermoelastic Phase}

This phase is characterized by $d \varepsilon^{\mathrm{pl}}=0$, so that Eq. (7) reads, using Eq. (5)

$$
d \sigma=\hat{C} d \varepsilon^{\mathrm{el}}=\hat{C}\left(d \varepsilon-d \varepsilon^{\mathrm{th}}\right)
$$

In our cylindrical case, it reads according to Eqs. (6) and (9)

$$
\begin{gathered}
d \sigma_{r r}=\left(\frac{4 G}{3}+K\right) d \varepsilon_{r r}+\left(K-\frac{2 G}{3}\right) d \varepsilon_{\theta \theta}-3 K \alpha d T \\
d \sigma_{\theta \theta}=\left(\frac{4 G}{3}+K\right) d \varepsilon_{\theta \theta}+\left(K-\frac{2 G}{3}\right) d \varepsilon_{r r}-3 K \alpha d T \\
d \sigma_{z z}=\left(K-\frac{2 G}{3}\right)\left(d \varepsilon_{r r}+d \varepsilon_{\theta \theta}\right)-3 K \alpha d T
\end{gathered}
$$

We are especially interested in studying concrete layer behavior Thus, a dimensionless radius-thickness ratio $\rho=\left(r-R_{0}\right) / e$ is introduced where $R_{0}$ and $e=$ internal radius and the thickness of the concrete wall. This dimensionless number allows us to neglect $\varepsilon_{\theta \theta}$ with respect to $\varepsilon_{r r}$ and $\sigma_{r r}$ with respect to $\sigma_{\theta \theta}$. Indeed, according to Eq. (11), $\left|\varepsilon_{\theta \theta} / \varepsilon_{r r}\right|=\mid \varepsilon_{\theta \theta} /\left[\varepsilon_{\theta \theta}+\left(R_{0} / e+\rho\right) \partial \varepsilon_{\theta \theta} / \partial \rho\right]$ $\lll 1$ because $R_{0} / e \ggg 1$.

In the same way, and according to Eq. (12), $\left|\sigma_{r n} / \sigma_{\theta \theta}\right|$ $=\left|\sigma_{r r} /\left[\sigma_{r r}+\left(R_{0} / e+\rho\right) \partial \sigma_{r r} / \partial \rho\right]\right| \lll 1$.

Then, in the following, it can be supposed that

$$
\sigma_{r r} \approx 0 \text { and } \varepsilon_{\theta \theta} \approx 0
$$

This approximation is foreseeable because when $R_{0} / e \ggg 1$, the problem tends locally toward a Cartesian geometry (in which $\sigma_{x x}=0$ and $\varepsilon_{y y}=0$ )

As a consequence of approximation (14), the radial strain variation can be expressed analytically as

$$
d \varepsilon_{r r}=\frac{9 K}{4 G+3 K} \alpha d T=\frac{1+\nu}{1-\nu} \alpha d T
$$

and then axial and orthoradial stress variations read

$$
d \sigma_{z z} \approx d \sigma_{\theta \theta} \approx-\frac{18 G K}{4 G+3 K} \alpha d T=-\frac{E_{0}}{1-\nu} \alpha \xi_{\mathrm{eq}}(T) d T
$$

The two last differential equations can be integrated on temperature

$$
\begin{gathered}
\varepsilon_{r r}=\frac{1+v}{1-v} \alpha\left(T-T_{0}\right) \\
\sigma_{z z} \approx \sigma_{\theta \theta} \approx-\frac{E_{0}}{1-v} \alpha \int_{T_{0}}^{T} \xi_{\mathrm{eq}}(T) d T
\end{gathered}
$$

However, the last equalities (15) and (16) are valid if there is no plastic flow:

- $f(\sigma, \xi)<0$ : elastic domain;

- $f=0$ and $d f<0$ : elastic unloading.

In the following, the conditions to reach the plastic criterion are studied. In our case, the stress tensor can be replaced by a vector

$$
\left(\sigma_{r r}, \sigma_{\theta \theta}, \sigma_{z z}\right)=\left(0, \sigma_{z z}, \sigma_{z z}\right)
$$

Then, the invariants of William-Warnke criterion (3) are

$$
\tau=-\frac{\sigma_{z z}}{\sqrt{3}}, \quad \sigma=\frac{2 \sigma_{z z}}{3}
$$

and

$$
\cos (\Theta)=1 \Rightarrow \delta(\Theta)=\delta(0)=\delta_{t}
$$

Then 


$$
\begin{aligned}
d f & =-\frac{1}{3}\left(\sqrt{3}-2 \delta_{t}\right) d \sigma_{z z}-\delta_{t} \rho_{0} d \xi \\
& =\left[\left(\sqrt{3}-2 \delta_{t}\right) \frac{E_{0}}{3(1-v)} \alpha \xi_{\text {eq }}(T)-\delta_{t} \rho_{0} \frac{\partial \xi_{\text {eq }}}{\partial T}\right] d T \geqslant 0
\end{aligned}
$$

which means that there is no elastic unloading when temperature increases. Thus, the only case in the elastic phase is when the loading stays in the elastic domain $(f<0)$, which is verified when

$$
\left|\sigma_{z z}\right|=-\sigma_{z z} \leqslant \frac{3 \rho_{0} \delta_{t}}{-2 \delta_{t}+\sqrt{3}} \xi_{\mathrm{eq}}(T)
$$

The last result is foreseeable because $\left[3 \rho_{0} \delta_{t} /\left(-2 \delta_{t}+\sqrt{3}\right)\right] \xi_{\mathrm{eq}}(T)$ $=f_{\text {bc }} \xi_{\text {eq }}(T)$, which is the bicompressive elastic limit after dehydration. The stresses are, indeed, bicompressive since they are concentrated in the plane $(\theta, z)$.

The combination of equality (16) and inequality (17) on $\sigma_{z z}$ allows us to deduce a critical temperature of plastification $T_{\mathrm{pl}}$ at which a plastic flow starts. $T_{\mathrm{pl}}$ verifies

$$
\frac{E_{0}}{1-\nu} \alpha \int_{T_{0}}^{T_{\mathrm{pl}}} \xi_{\mathrm{eq}}(T) d T \approx f_{\mathrm{bc}} \xi_{\mathrm{eq}}\left(T_{\mathrm{pl}}\right)
$$

$\xi_{\text {eq }}$ varies linearly on temperature (for $T<773 \mathrm{~K}$ ) following the function

$$
\xi_{\text {eq }}(T)=1-\frac{0.83}{500}\left(T-T_{0}\right)
$$

For this curve of dehydration, the resolution of Eq. (18) gives the "plastification" temperature $T_{\mathrm{pl}} \approx 440 \mathrm{~K}$ and then the dehydration degree $\xi_{\mathrm{pl}}=\xi_{\text {eq }}\left(T_{\mathrm{pl}}\right) \approx 0.75$.

\section{Plastic Phase}

Now, it is supposed that plastification temperature $T_{\mathrm{pl}}$ is exceeded. There is a plastic flow and according to Eq. (8)

$$
d \varepsilon_{r r}^{\mathrm{pl}}=d \lambda \frac{\partial f}{\partial \sigma_{r r}}, \quad d \varepsilon_{\theta \theta}^{\mathrm{pl}}=d \lambda \frac{\partial f}{\partial \sigma_{\theta \theta}}, \quad \text { and } d \varepsilon_{z z}^{\mathrm{pl}}=d \lambda \frac{\partial f}{\partial \sigma_{z z}}
$$

where using flow rule (3), for $(i=r, \theta, z)$

$$
\frac{\partial f}{\partial \sigma_{i i}}=\frac{s_{i i}}{2 \tau}+\left(\sigma-\rho_{0} \xi\right) P_{i}+\frac{1}{3} \delta(\Theta)
$$

with

$$
P_{i}=-\frac{d \delta}{d \Theta} \frac{1}{2 \sqrt{12} \tau^{3}}\left[\left(2 \sigma_{1}^{\mathrm{pr}}-\sigma_{2}^{\mathrm{pr}}-\sigma_{3}^{\mathrm{pr}}\right) s_{i i}+a_{i} \tau^{2}\right]
$$

where $\sigma_{1}^{\mathrm{pr}}, \sigma_{2}^{\mathrm{pr}}$ and $\sigma_{3}^{\mathrm{pr}}=$ principal stresses; and $a_{i}=-4$ if $\sigma_{i i}=\sigma_{1}^{\mathrm{pr}}$ and $a_{i}=2$ otherwise.

Approximation (14) is used again: $\varepsilon_{\theta \theta} \approx 0$ and $\sigma_{r r} \approx 0$, and so

$$
\sigma_{\theta \theta} \approx \sigma_{z z}
$$

Knowing that $\sigma_{\theta \theta} \approx \sigma_{z z}<0 \approx \sigma_{r r}$, it can be concluded that $\sigma_{r r}=\sigma_{1}^{\mathrm{pr}}$ and then $a_{r}=-4$ and $a_{\theta}=a_{z}=2$. Finally

$$
\frac{\partial f}{\partial \sigma_{r r}}=\frac{1}{\sqrt{3}}+\frac{\delta_{t}}{3}=0.835
$$

and

$$
\frac{\partial f}{\partial \sigma_{z z}}=\frac{\partial f}{\partial \sigma_{\theta \theta}}=-\frac{1}{2 \sqrt{3}}+\frac{\delta_{t}}{3}=-0.0304
$$

A first conclusion is

$$
d \varepsilon_{\theta \theta}^{\mathrm{pl}}=d \varepsilon_{z z}^{\mathrm{pl}}
$$

and

$$
d \varepsilon_{r r}^{\mathrm{pl}}=-\frac{\frac{1}{\sqrt{3}}+\frac{\delta_{t}}{3}}{-\frac{1}{2 \sqrt{3}}+\frac{\delta_{t}}{3}} d \varepsilon_{\theta \theta}^{\mathrm{pl}}=-27.47 d \varepsilon_{\theta \theta}^{\mathrm{pl}}
$$

It is clear then that the most important plastic strain is done in the radial direction.

In our cylindrical case, thermoelastic state equation (7), taking into account plastic strain rate, reads

$$
\begin{aligned}
d \sigma_{r r}= & \left(\frac{4 G}{3}+K\right)\left(d \varepsilon_{r r}-d \varepsilon_{r r}^{\mathrm{pl}}\right)+\left(K-\frac{2 G}{3}\right)\left(d \varepsilon_{\theta \theta}-d \varepsilon_{\theta \theta}^{\mathrm{pl}}\right) \\
& -\left(K-\frac{2 G}{3}\right) d \varepsilon_{z z}^{\mathrm{pl}}-3 K \alpha d T \\
d \sigma_{\theta \theta}= & \left(\frac{4 G}{3}+K\right)\left(d \varepsilon_{\theta \theta}-d \varepsilon_{\theta \theta}^{\mathrm{pl}}\right)+\left(K-\frac{2 G}{3}\right)\left(d \varepsilon_{r r}-d \varepsilon_{r r}^{\mathrm{pl}}\right) \\
& -\left(K-\frac{2 G}{3}\right) d \varepsilon_{z z}^{\mathrm{pl}}-3 K \alpha d T \\
d \sigma_{z z}= & -\left(\frac{4 G}{3}+K\right) d \varepsilon_{z z}^{\mathrm{pl}}+\left(K-\frac{2 G}{3}\right)\left(d \varepsilon_{r r}-d \varepsilon_{r r}^{\mathrm{pl}}+d \varepsilon_{\theta \theta}-d \varepsilon_{\theta \theta}^{\mathrm{pl}}\right) \\
& -3 K \alpha d T
\end{aligned}
$$

Still using approximations $(14), \varepsilon_{\theta \theta} \approx 0, \sigma_{r r} \approx 0$ leading then to $\sigma_{\theta \theta} \approx \sigma_{z z}$ and $\varepsilon_{\theta \theta}^{\mathrm{pl}} \approx \varepsilon_{z z}^{\mathrm{pl}}$ allows us to establish the following relation:

$$
d \sigma_{z z}=-\frac{18 G K}{4 G+3 K}\left(d \varepsilon_{\theta \theta}^{\mathrm{pl}}+\alpha d T\right)
$$

Because the plastification temperature $T_{\mathrm{pl}}$ is exceeded, it can be affirmed according to Eq. (17) that at each time (in the plasticized zone)

$$
\sigma_{z z}=\frac{3 \rho_{0} \delta_{t}}{2 \delta_{t}-\sqrt{3}} \xi_{\mathrm{eq}}(T)=-f_{\mathrm{bc}} \xi_{\mathrm{eq}}(T)
$$

It can be deduced now from Eqs. (19), (21), and (22) that

$$
d \varepsilon_{\theta \theta}^{\mathrm{pl}}=d \varepsilon_{z z}^{\mathrm{pl}}=\frac{4 G+3 K}{18 G K} f_{\mathrm{bc}} d \xi_{\mathrm{eq}}-\alpha d T=\frac{(1-v) f_{\mathrm{bc}}}{E_{0}} \frac{d \xi_{\mathrm{eq}}}{\xi_{\mathrm{eq}}}-\alpha d T
$$

and then

$$
\begin{aligned}
d \varepsilon_{r r}^{\mathrm{el}} & =d \varepsilon_{r r}-d \varepsilon_{r r}^{\mathrm{pl}}=\frac{1}{4 G+3 K}\left[(6 K-4 G) d \varepsilon_{\theta \theta}^{\mathrm{pl}}+9 K \alpha d T\right] \\
& =\frac{2 \nu f_{\mathrm{bc}}}{E_{0}} \frac{d \xi_{\mathrm{eq}}}{\xi_{\mathrm{eq}}}+\alpha d T
\end{aligned}
$$

The first differential equality (23) can be integrated between the plastification temperature $T_{\mathrm{pl}}\left[\varepsilon_{\theta \theta}^{\mathrm{pl}}\left(T_{\mathrm{pl}}\right)=\varepsilon_{z z}^{\mathrm{pl}}\left(T_{\mathrm{pl}}\right)=0\right]$ and the actual temperature $T$. 
T(K) Temperazure of heated surface

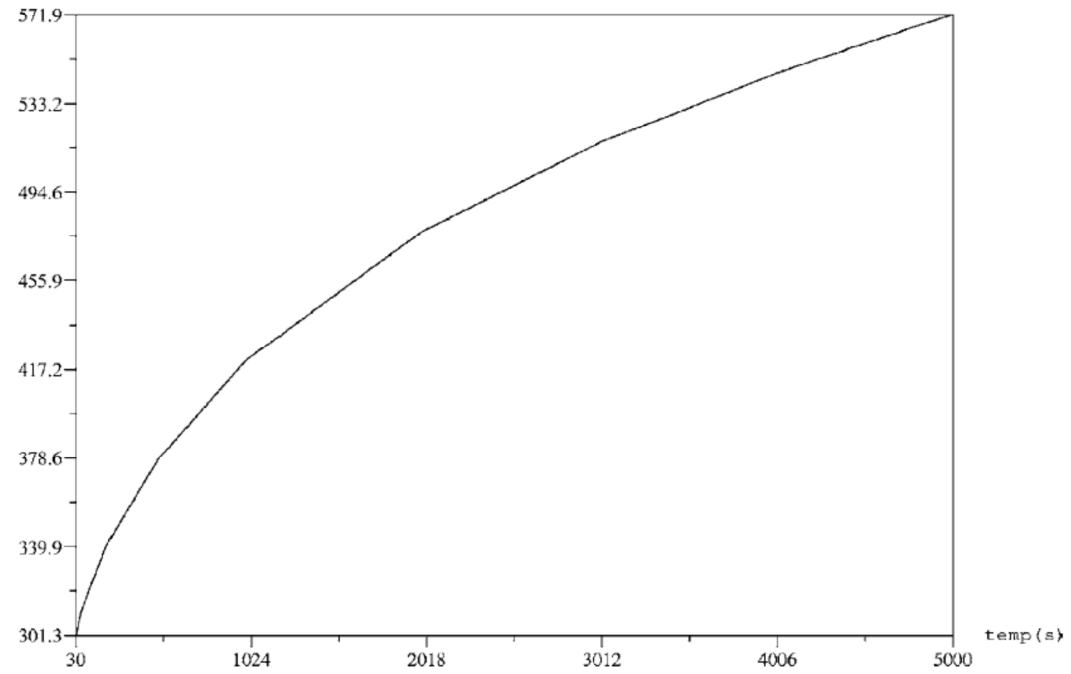

Fig. 2. Temperature of the heated surface (intrados) $T_{\mathrm{pl}}$ is reached after $1 \times 10^{3} \mathrm{~s}$

$$
\varepsilon_{\theta \theta}^{\mathrm{pl}}=\varepsilon_{z z}^{\mathrm{pl}}=\frac{(1-v) f_{\mathrm{bc}}}{E_{0}} \log \left(\frac{\xi_{\mathrm{eq}}(T)}{\xi_{\mathrm{pl}}}\right)-\alpha\left(T-T_{\mathrm{pl}}\right)
$$

The radial plastic strain (which is more important than the orthoradial or axial plastic strain) can be then expressed from Eq. (19)

$$
\begin{aligned}
\varepsilon_{r r}^{\mathrm{pl}}=- & \frac{\frac{1}{\sqrt{3}}+\frac{\delta_{t}}{3}}{-\frac{1}{2 \sqrt{3}}+\frac{\delta_{t}}{3}} \varepsilon_{\theta \theta}^{\mathrm{pl}} \\
=- & \frac{\frac{1}{\sqrt{3}}+\frac{\delta_{t}}{3}}{-\frac{1}{2 \sqrt{3}}+\frac{\delta_{t}}{3}}\left[\frac{(1-v) f_{\mathrm{bc}}}{E_{0}} \log \left(\frac{\xi_{\mathrm{eq}}(T)}{\xi_{\mathrm{pl}}}\right)-\alpha\left(T-T_{\mathrm{pl}}\right)\right]
\end{aligned}
$$

The integration of the second differential equation (24) between $T_{\mathrm{pl}}$ and $T$ needs to know the elastic radial strain when the plastification temperature $T_{\mathrm{pl}}$ is reached. The value $\varepsilon_{r r}^{\mathrm{el}}\left(T_{\mathrm{pl}}\right)$ is the value of $\varepsilon_{r r}^{\mathrm{el}}$ when plasticity is just beginning and is, therefore, given by the expression of elastic phase (15)

$$
\varepsilon_{r r}^{\mathrm{el}}\left(T_{\mathrm{pl}}\right)=\varepsilon_{r r}\left(T_{\mathrm{pl}}\right)=\frac{1+v}{1-v} \alpha\left(T_{\mathrm{pl}}-T_{0}\right)
$$

and then

$$
\varepsilon_{r r}^{\mathrm{el}}=\frac{1+v}{1-\nu} \alpha\left(T_{\mathrm{pl}}-T_{0}\right)+\frac{2 v f_{\mathrm{bc}}}{E_{0}} \log \left(\frac{\xi_{\mathrm{eq}}(T)}{\xi_{\mathrm{eq}}\left(T_{\mathrm{pl}}\right)}\right)+\alpha\left(T-T_{\mathrm{pl}}\right)
$$

Finally, the total radial strain $\varepsilon_{r r}=\varepsilon_{r r}^{\mathrm{el}}+\varepsilon_{r r}^{\mathrm{pl}}$ can be deduced from Eqs. (26) and (27). The elastic orthoradial and axial strains can also be expressed

$$
\varepsilon_{\theta \theta}^{\mathrm{el}}=\varepsilon_{z z}^{\mathrm{el}}=-\varepsilon_{\theta \theta}^{\mathrm{pl}}=-\varepsilon_{z z}^{\mathrm{pl}}
$$

from Eq. (25)

- In Fig. 2 is presented the temperature of a heated surface. I can be noticed that the plastification temperature is reached after $1 \times 10^{3} \mathrm{~s}$. At the same moment, the radial plastic flow starts (Fig. 3) and the variation of the axial compressive stress changes (Fig. 4). Fig. 5 also shows that the slope of the total radial strain changes due to the plastic flow.

- It can also be noticed in Figs. 3-5 that there is good agreement between the analytical curves [simplified approach using approximation (14)] and the numerical ones (complete model without any approximation). The approximations introduced before $\left(\sigma_{r r} \approx 0\right.$ and $\left.\varepsilon_{\theta \theta} \approx 0\right)$ are then validated.

\section{Explanation of Thermomechanical Spalling}

In the following, the results of the previous section are used to explain under which conditions thermomechanical spalling can occur.

\section{General Case Taking Account of Decohesion} and Softening

Layers of concrete having a temperature greater than $T_{\mathrm{pl}}$ are in a plastification process. The remaining layers are still in an elastic phase. Let us note the $r_{\mathrm{pl}}$ radius where $T=T_{\mathrm{pl}}$. For $r>r_{\mathrm{pl}}$, it is the elastic domain: $T<T_{\mathrm{pl}}$. From Eq. (16), the axial stress can be expressed

$$
\sigma_{z z}=-\left[E_{0} /(1-v)\right] \alpha \int_{T_{0}}^{T} \xi_{\mathrm{eq}}(T) d T
$$

and then 


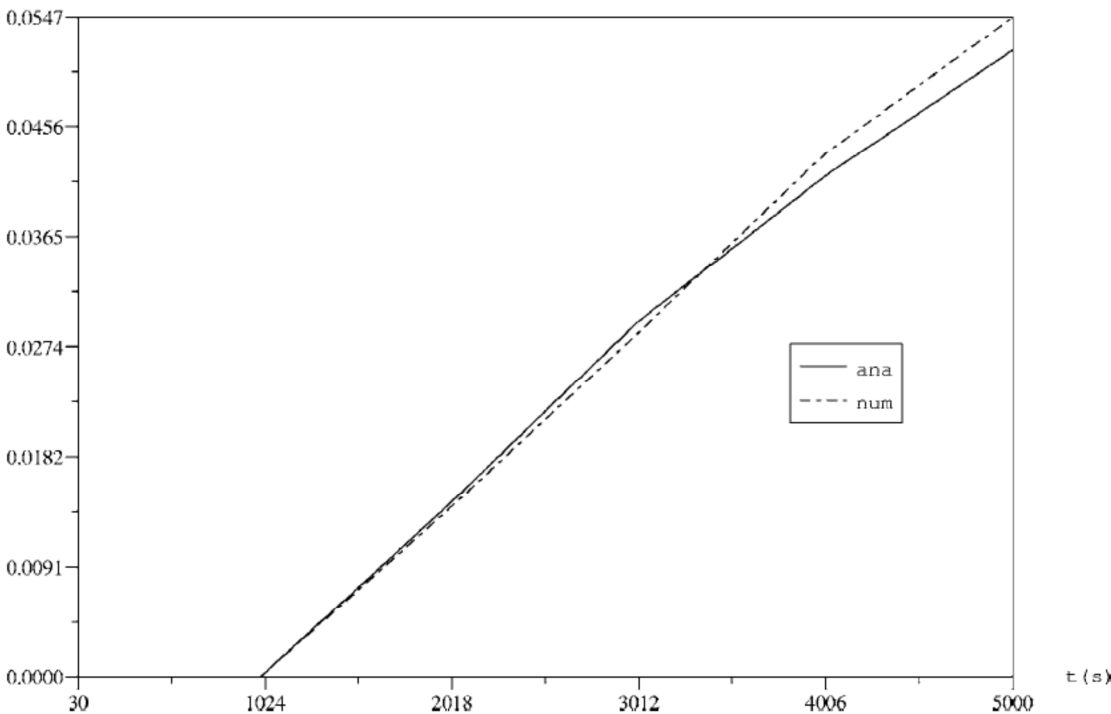

Fig. 3. Plastic radial strain at heated surface: existence of two phases; the plastic phase starts when $T_{\mathrm{pl}}$ is reached (Fig. 2)

$$
\frac{\partial \sigma_{z z}}{\partial r}=\frac{\partial \sigma_{z z}}{\partial T} \frac{\partial T}{\partial r}=-\frac{E_{0}}{1-v} \alpha \xi_{\mathrm{eq}}(T) \frac{\partial T}{\partial r}>0
$$

For $r<r_{\mathrm{pl}}$, it is the plastic domain: $T>T_{\mathrm{pl}}$. From Eq. (22), $\sigma_{z z}=-\xi_{\mathrm{eq}}(T) f_{\mathrm{bc}}$ and then

$$
\frac{\partial \sigma_{z z}}{\partial r}=\frac{\partial \sigma_{z z}}{\partial T} \frac{\partial T}{\partial r}=-f_{\mathrm{bc}} \frac{\partial \xi_{\mathrm{eq}}}{\partial T} \frac{\partial T}{\partial r}<0
$$

It has been just proven that $\sigma_{z z}$ (and also $\sigma_{\theta \theta}$ ) changes of variation at $r=r_{\mathrm{pl}}$. There is a plug of axial and orthoradial stresses at

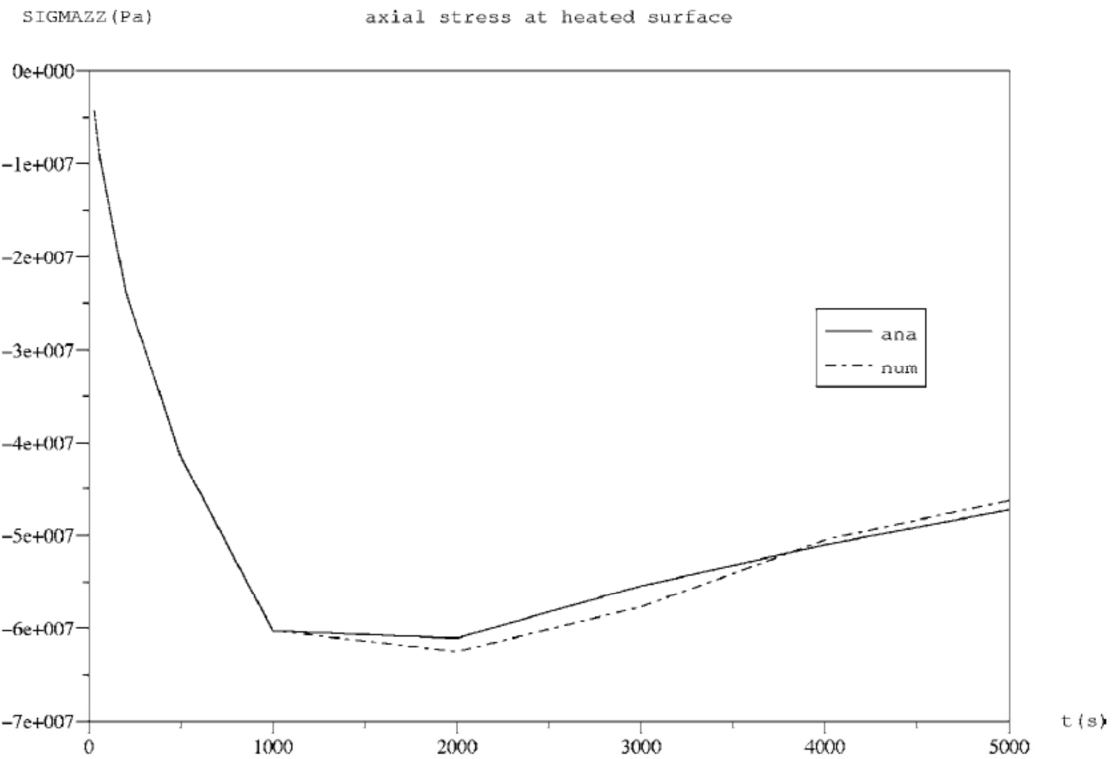

Fig. 4. Compressive axial stress at heated surface: variation changes when $T=T_{\mathrm{pl}}$ 


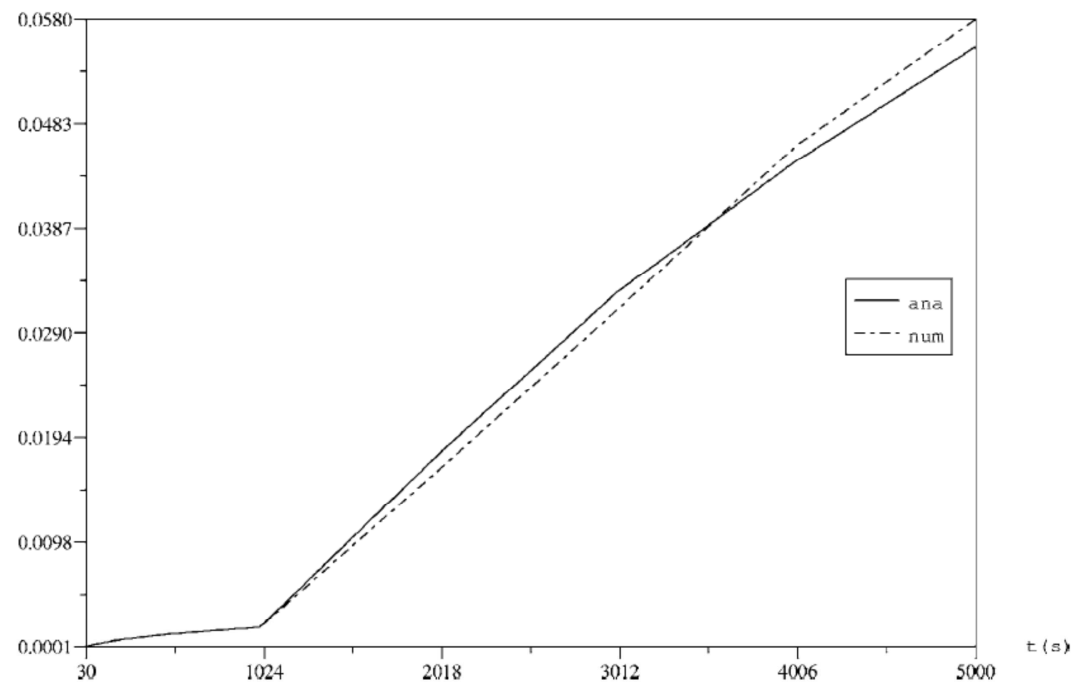

Fig. 5. Total radial strain at heated surface: slope changes when $T=T_{\mathrm{pl}}$

$r=r_{\mathrm{pl}}$ (Fig. 6) and this change of slope may explain spalling by failure in the plane $(\theta, z)$

Thus, the problem of mechanical spalling is brought back to study the propagation of the plasticized zone by the mean of $r_{\mathrm{pt}}$ where the temperature reaches $T_{\mathrm{pl}}$. Forecasting the spalling can be, therefore, reduced to a purely thermal problem.
What follows is a study of the different parts of the constitutive model to examine which term is necessary to forecast the spalling.

\section{Neglecting Thermal Softening}

- $E(T)=E_{0}$ instead of expression (1).

SIGMAZZ (Pa) axial stress after $1 \mathrm{~h} 30$

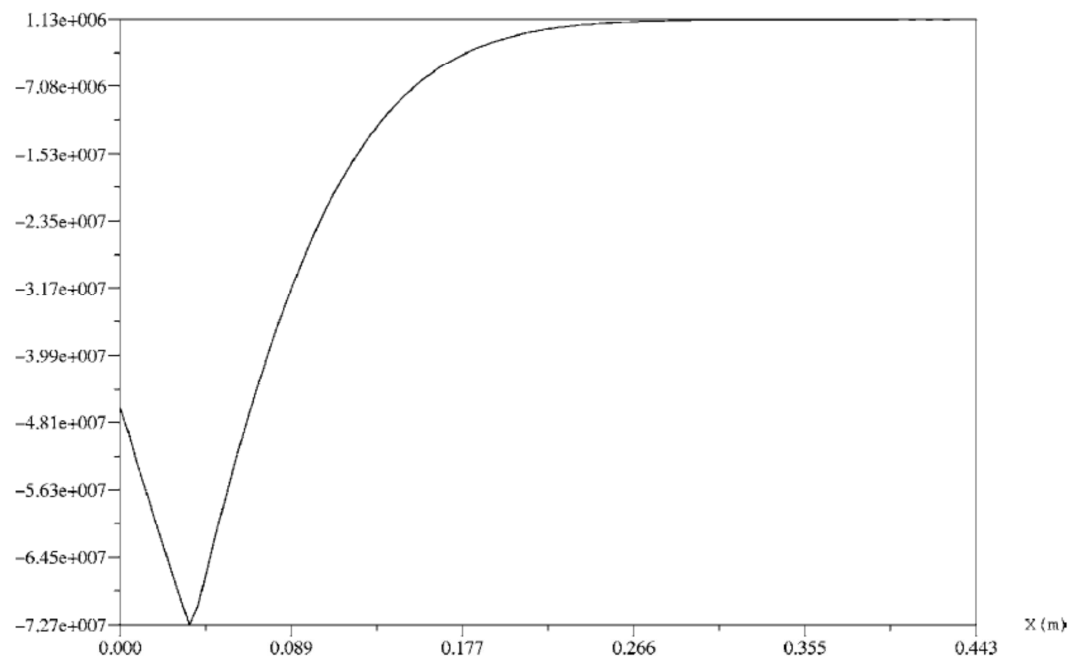

Fig. 6. Axial stress after $1 \mathrm{~h} 30 \mathrm{~min}$ of heating: existence of a stress "plug" and a change of slope at the surface $r=r_{\mathrm{pl}}$, which may explain failure at this surface 
- During the elastic phase, the expression of radial strain is still $\varepsilon_{r r}=[(1+v) /(1-v)] \alpha\left(T-T_{0}\right)$. However, expression (16) of axial stress reads

$$
\sigma_{z z} \approx \sigma_{\theta \theta} \approx-\frac{E_{0}}{1-v} \alpha\left(T-T_{0}\right)
$$

Eq. (18) for determining the plastic temperature changes into

$$
\frac{E_{0}}{1-\nu} \alpha\left(T_{\mathrm{pl}}-T_{0}\right) \approx f_{\mathrm{bc}} \xi_{\mathrm{eq}}\left(T_{\mathrm{pl}}\right)
$$

- During the plastic phase, $\sigma_{z z}=-f_{\text {bc }} \xi_{\text {eq }}(T)$ is still valid but the different strains change into

$$
\begin{gathered}
\varepsilon_{\theta \theta}^{\mathrm{pl}}=\varepsilon_{z z}^{\mathrm{pl}}=\frac{(1-v) f_{\mathrm{bc}}}{E_{0}}\left[\xi_{\mathrm{eq}}(T)-\xi_{\mathrm{pl}}\right]-\alpha\left(T-T_{\mathrm{pl}}\right) \\
\varepsilon_{r r}^{\mathrm{pl}}=-\frac{\frac{1}{\sqrt{3}}+\frac{\delta_{t}}{3}}{-\frac{1}{2 \sqrt{3}}+\frac{\delta_{t}}{3}} \varepsilon_{\theta \theta}^{\mathrm{pl}} \\
\varepsilon_{r r}^{\mathrm{el}}=\frac{1+v}{1-v} \alpha\left(T_{\mathrm{pl}}-T_{0}\right)+\frac{2 v f_{\mathrm{bc}}}{E_{0}}\left[\xi_{\mathrm{eq}}(T)-\xi_{\mathrm{pl}}\right]+\alpha\left(T-T_{\mathrm{pl}}\right)
\end{gathered}
$$

In this case, the spalling explanation is always valid, because $\partial \sigma_{z z} / \partial r$ is still positive for $r<r_{\mathrm{pl}}$ according to Eq. (22) and negative when $r>r_{\mathrm{pl}}$ according to Eq. (28).

It can be noticed that the plastification temperature is lower in this case $\left(T_{\mathrm{pl}} \approx 425 \mathrm{~K}\right.$ instead of $\left.440 \mathrm{~K}\right)$. This means that the plastification radius $r_{\mathrm{pl}}$ is higher.

\section{Neglecting Thermal Decohesion}

In this section, plasticity criterion (3) is independent of the dehydration degree $\xi[f(\sigma)$ instead of $f(\sigma, \xi)]$; the strength diminution caused by dehydration is neglected.

For the elastic phase, the expressions of strains and stresses are unchanged. However, the plastification temperature changes and is given while solving

$$
\frac{E_{0}}{1-\nu} \alpha \int_{T_{0}}^{T_{\mathrm{pl}}} \xi_{\mathrm{eq}}(T) d T \approx f_{\mathrm{bc}}
$$

During the plastic phase, the axial stress is constant

$$
\sigma_{z z}=-f_{\mathrm{bc}}
$$

Then, the different strains change into

$$
\begin{gathered}
\varepsilon_{\theta \theta}^{\mathrm{pl}}=\varepsilon_{z z}^{\mathrm{pl}}=-\alpha\left(T-T_{\mathrm{pl}}\right) \\
\varepsilon_{r r}^{\mathrm{pl}}=-\frac{\frac{1}{\sqrt{3}}+\frac{\delta_{t}}{3}}{-\frac{1}{2 \sqrt{3}}+\frac{\delta_{t}}{3}} \varepsilon_{\theta \theta}^{\mathrm{pl}}=\frac{\frac{1}{\sqrt{3}}+\frac{\delta_{t}}{3}}{-\frac{1}{2 \sqrt{3}}+\frac{\delta_{t}}{3}} \alpha\left(T-T_{\mathrm{pl}}\right) \\
\varepsilon_{r r}^{\mathrm{el}}=\frac{1+v}{1-v} \alpha\left(T_{\mathrm{pl}}-T_{0}\right)+\alpha\left(T-T_{\mathrm{pl}}\right)
\end{gathered}
$$

The spalling explanation is not valid in this case because $\partial \sigma_{z z} / \partial r$ is null for $r<r_{\mathrm{pl}}$ according to Eq. (29). There is no plug of axial and orthoradial stresses able to explain a failure in the plane $(\theta, z)$. It means that taking into account the thermal decohesion is necessary to be able to forecast the spalling.

\section{Neglecting Both Thermal Decohesion and Softening}

Neglecting both thermal decohesion and softening means that the chemomechanical couplings are neglected $\left[f(\underline{\sigma}), E(T)=E_{0}\right]$. The stress expressions are obtained directly from the last case [by simply replacing $\xi_{\text {eq }}(T)$ by 1$]$. The plastic temperature changes and verifies

$$
\frac{E_{0}}{1-\nu} \alpha\left(T_{\mathrm{pl}}-T\right) \approx f_{\mathrm{bc}}
$$

Like the last case, the compressive stress is constant in the plasticized zone near the heated surface. The spalling cannot be explained in this case. There is rather a crushing failure mode at the surface.

\section{Conclusion}

In this paper, the approximative expressions of compressive stresses and strains (elastic, plastic, and total) have been established in both elastic and plastic phases. The existence of a plastification temperature has also been obtained. Then, the spalling phenomenon is related to a "peak" appearing on the stresses at a certain depth $r_{\mathrm{pl}}$ where the plastification temperature is attained The existence of this peak was studied in different cases (with or without thermal softening and decohesion) to conclude that it is the thermal decohesion which is necessary to forecast the thermal spalling. The plastification temperature, obtained just while treating a simple problem of thermal conduction, is useful in identifying the plasticized zone where a spalling process is probable.

\section{Notation}

The following symbols are used in this paper

$C=$ volumic heat capacity $\left(\mathrm{J} \mathrm{K}^{-1} \mathrm{~m}^{-3}\right)$

$E=$ Young modulus $(\mathrm{Pa})$;

$e=$ thickness $(\mathrm{m})$;

$f_{\mathrm{bc}}=$ bicompressive elastic limit $(\mathrm{Pa})$

$f_{c}=$ compressive elastic limit $(\mathrm{Pa})$;

$G=$ shear modulus $(\mathrm{Pa})$

$K=$ bulk modulus $(\mathrm{Pa})$;

$R_{0}=$ internal radius $(\mathrm{m})$; and

$T=$ temperature $(\mathrm{K})$

$\alpha=$ thermal dilation coefficient $\left(\mathrm{K}^{-1}\right)$;

$\chi=$ dehydration degree;

$\kappa=$ conductivity $\left(\mathrm{J} \mathrm{s}^{-1} \mathrm{~m}^{-1} \mathrm{~K}^{-1}\right)$;

$v=$ Poisson ratio;

$\rho=$ cohesion pressure $(\mathrm{Pa})$;

$\sigma=\operatorname{stress}(\mathrm{Pa})$

$\tau=$ characteristic time (s); and

$\varepsilon=$ strain.

\section{Superscripts}

el = elastic;

$\mathrm{pl}=$ plastic;

$\mathrm{pr}=$ principal

th $=$ thermal; and

tot $=$ total. 


\section{Subscripts}

$$
\begin{aligned}
0 & =\text { initial; } \\
\theta & =\text { orthoradial; } \\
\text { eq } & =\text { equilibrium; } \\
h & =\text { hydrates; } \\
r & =\text { radial; and } \\
z & =\text { axial. }
\end{aligned}
$$

\section{References}

Anderberg, Y. (1997). "Spalling phenomenon of HPC and Oc." Proc., Int. Workshop on Fire Performance of High-Strength Concrete, NIST Special Publication 919, L. T. Phan, N. J. Carino, D. Duthinh, and E. Garboczi, eds., National Institute of Standards and Technology, Gaithersburg, Md., 69-73.

Bazant, Z. P. (1997). "Analysis of pore pressure, thermal stresses, and fracture in rapidly heated concrete." Proc., Int. Workshop on Fire Performance of High-Strength Concrete, NIST Special Publication 919, L. T. Phan, N. J. Carino, D. Duthinh, and E. Garboczi, eds., National Institute of Standards and Technology, Gaithersburg, Md., $155-164$.

Fasseu, P. (1997). "Eurotunnel fire: Analysis of the effect of the fire on the concrete quality." Research Rep. No. 96.6002532, Laboratoire Régional des Ponts et Chaussées de Lille, Lille, France.

Ulm, F.-J., Acker, P., and Lévy, M. (1999a). "The 'Chunnel' fire. II Analysis of concrete damage." J. Eng. Mech., 125(3), 283-289.

Ulm, F.-J., Coussy, O., and Bazant, Z. P. (1999b). "The 'Chunnel' fire. I: Chemoplastic softening in rapidly heated concrete." J. Eng. Mech., $125(3), 272-282$.

William, K. J., and Warnke, E. P. (1975). "Constitutive model for the triaxial behavior of concrete." Proc., IABSE 19th Seminar on Concrete Structures Subjected to Triaxial Stresses, Paper III-1, International Association for Bridge and Structural Engineering, Zurich, Switzerland. 